\title{
ARCHITECTURE
}

\section{FEATURES OF ARTIFICIAL LIGHTING WHICH IS CONSISTENT WITH HUMAN BIORYTHMS}

\section{Lidiya Koval $^{1}$}

DOI: https://doi.org/10.30525/978-9934-588-11-2_22

One of the most important issues related to lighting is its impact on living organisms (humans in particular). Modern test and measurement methods make it possible to investigate the effects of light much more carefully, and with the new capabilities of LED technologies for colour temperature control and colour rendering these results are much easier to put into practice [8]. In general, the transition of society to digital LED technologies in lighting may be regarded as a paradigm shift that is happening at a rapid pace. It concerns not only visual perception and energy efficiency, but also the optimization of biological and emotional impact of light [7].

Various definitions, all of which have their nuances, are used to designate lighting that recognizes the effects of light on the body and human activity. In this context the term «biologically effective lighting» is used, but the adjective «biological» here also covers visual processes. The term «dynamic illumination» is widely used if the colour temperature and illuminance change according to daylight, affecting human circadian rhythms. However, the same term refers to light which changes (for example, in colour) but has no biological effect. The term «circadian lighting» is used when illumination is intended to correct or stabilize human circadian rhythms [8]. Over the past few years the notion «human centric lighting» has become particularly prevalent in terms of lighting that meets individual living and working conditions of a user at any time, satisfying not only the visual requirements but also affecting a person emotionally and biologically [7]. This study is based on the proposition that any lighting of a person's living environment is intended to meet his/her needs and therefore is focused on him/her. Thus, the appropriate artificial lighting (such that takes into account the effect of light on the body and human activity) is considered as lighting which is consistent with human biorhythms.

The true extent of the dependence of the human body on lighting has only become apparent in recent decades. The importance of research into the effects of light on a person's biological clock is indicated by the fact that in 2017 Jeffrey C. Hall, Michael Rosbash and Michael W. Young were awarded the Nobel Prize in Medicine for their work on the mechanism known as «circadian rhythm» [4]. Human circadian rhythm is synchronized with the solar daily cycle and regulates not only periods of activity and rest, as well as biorhythms, but also physiological functions at the cellular level.

${ }^{1}$ Kyiv National University of Construction and Architecture, Ukraine 
Today, it is known that through circadian rhythm light can affect both productivity and human health [6, p. 80-87]: seasonal decrease in daylight in winter in the extreme northern and southern latitudes may cause depression - seasonal affective disorder (SAD) or «winter blues»; daily alternation of light and darkness significantly affects the quality of sleep, extreme cases of which are diagnosed as delayed sleep phase syndrome.

Until recently, it was believed that light detection (photoreception) occurs exclusively with the help of retinal cells such as rods and cones. However, modern photobiologists have identified intrinsic photosensitive retinal ganglion cells (ipRGCs), which are most sensitive to blue light, with a peak of about 460-490 nm [1]. While rods and cones determine shape and colour, imparting this information to the visual cortex, ipRGCs detect irradiation and direct their information to many brain structures, including the suprachiasmatic nucleus hypothalamic which has been studied most thoroughly. Due to the «light and dark» signals coming to it, this nucleus controls the production of the hormone melatonin, which regulates the circadian rhythm. Thus, the light received by the eye is transformed into neural signals that pass through the optic nerve in two ways, one is visual, whereas the other is non-visual [3]. Therefore, when assessing the appropriateness of any method of lighting, one should take into account the physical properties of light, which differently affect the two biological systems of humans - visual and circadian (Fig. 1) [6, p. 85-87].

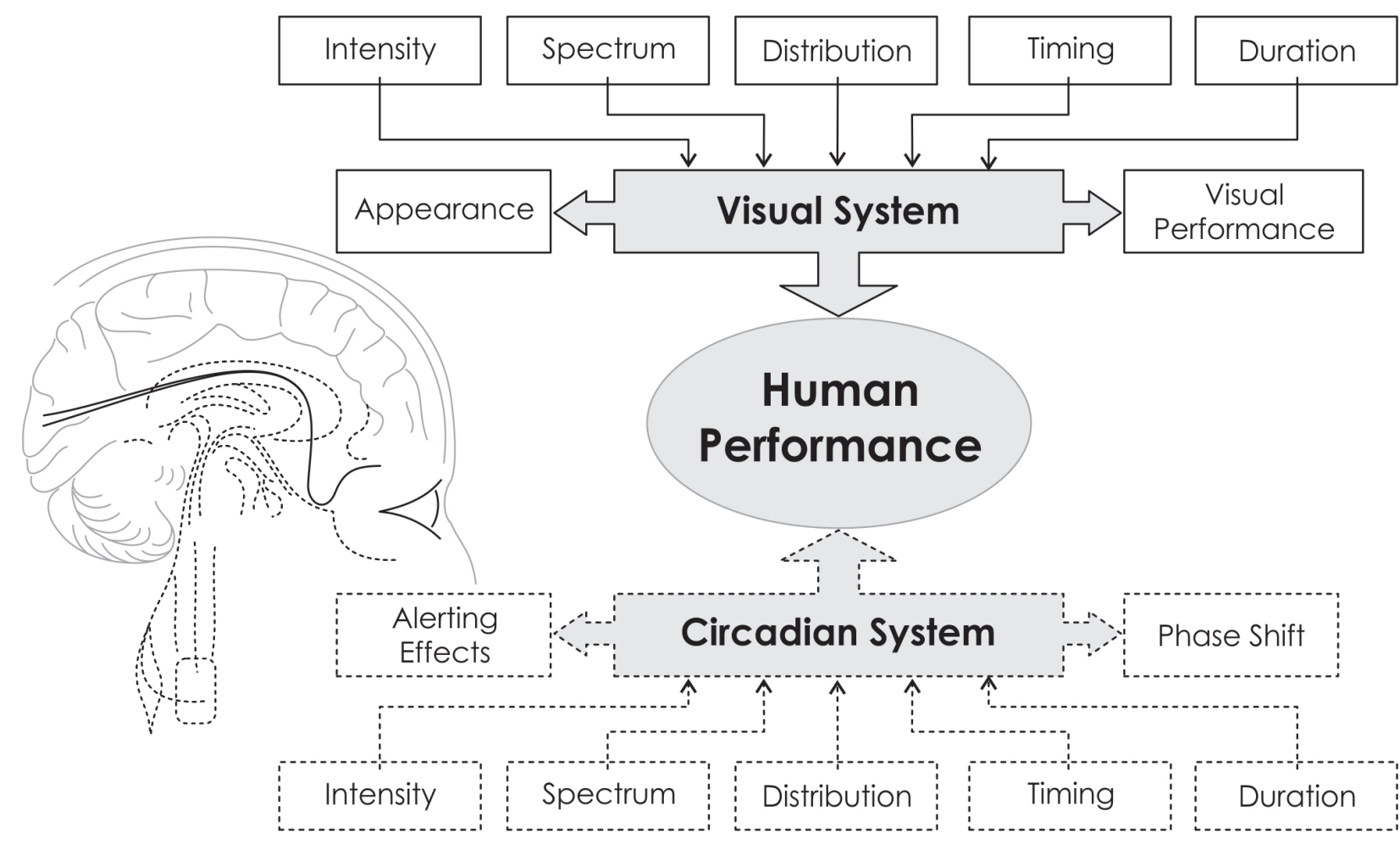

Figure 1. The influence of light on humans through the visual and circadian systems

Source: developed by the author [6, p. 80-81] 
Today, the main approach to quantifying the effect of artificial light on the circadian system is to calculate the effective amount of circadian stimulation by comparing the radiation spectrum of the source with the circadian action spectrum, which results in a certain level of melanopic lux. Two sources of the same illuminance may have different melanopic lux, which indicates the relative potential of the influence of these sources on the circadian system [1]. In order to maintain the circadian rhythm, it is important that the melanopic levels of morning and daytime lighting are higher than in the evening. To do this, illuminance on the eye of 300 to $500 \mathrm{~lx}$ with a colour temperature of $5500 \mathrm{~K}$. should be maintained for several morning hours. Two hours before bedtime illuminance on the eye of up to $50 \mathrm{~lx}$ is recommended with colour temperature of $2700-3000 \mathrm{~K}$ and minimized blue light components [7].

In addition to the general spectrum of the source and the intensity of the blue part in its radiation, the direction in which light enters the eye is important for the degree of influence of lighting on the circadian system. The field of view of a person indoors has an angular range of about $70^{\circ}$ below the line of sight and up to $55^{\circ}$ above the line of sight. In most cases, the gaze is directed downwards, since the visual task is usually concentrated at the bottom of the field of view. As lighting within a visual task should first and foremost meet the visual requirements, it is not recommended to plan additional lighting for biological impact in this area. The sensitivity of photoreceptors to biological light effects is higher in the lower retina, so the range of the highest biological light efficiency is $-15^{\circ}-45^{\circ}$ to the line of sight [7].

Over the past few years the lighting industry has been focused on creating products that can positively influence the human circadian rhythm (for example, [5]). Such lighting systems tend to use high light stimulation in the morning and limit it a few hours before bedtime [1]. Practical validation of the provisions for biologically effective and human centric lighting at the level of implemented projects mainly concerns health care facilities, including homes for the elderly [2], and school premises. Since natural light is traditionally considered to be the major contribution to the comfortable well-being of a person, the better artificial lighting imitates daylight, the more pleasant and perfect it is felt by humans [4]. In this regard, an effective method of maintaining circadian rhythm is to optimize the levels of natural light, in cases where it is insufficient, by supplementing it with artificial lighting sources [2]. The use of LEDs for such lighting provides the necessary light spectrum and easy running by means of intelligent control systems [8].

Therefore, artificial lighting consistent with human biorhythms, has the following features: dynamism in determining the appropriate time of action, because the biological non-visual impact of light is most effective in the morning and can be detrimental to the sleep-wake cycle in the evening; increasing the light levels and presence of the blue part of the spectrum $(460-490 \mathrm{~nm})$ in the morning; reducing light levels and minimizing the blue part of the spectrum in the evening, especially several hours before bedtime; the biologically effective direction in which light enters the user's field of view is $-15^{\circ}-45^{\circ}$ to the line of sight. 


\section{References:}

1. Aurelien David (2019). Circadian-Friendly Light Emitters: From CCT-Tuning to Blue-Free Technologyp. LED professional Review, Issue 71, pp. 58-63.

2. Chien Szu-Cheng (2019). Implications for Human-Centric Lighting Design in Tropical Nursing Homes: A Pilot Study. LED professional Review, Issue 71, pp. 40-47.

3. CIE (2017). CIE Calls for Focused Research Efforts to Support Healthful Lighting Recommendations. LED professional. Retrieved from: https://www.led-professional.com/resources1/articles/cie-calls-for-focused-research-efforts-to-support-healthful-lighting-recommendations (accessed 9 August 2019).

4. Haumer Peter (2019). Healthy Light - LED Technology for Health and Care Applications. LED professional Review, Issue 71, pp. 68-73.

5. Lumileds (2019). Lumileds Enables Next Step in Human Centric Lighting with Launch of Luxeon Fusion. LED professional. Retrieved from: https://www.led-professional.com/products/ledmodules-led-light-engines/lumileds-enables-next-step-in-human-centric-lighting-with-launch-ofluxeon-fusion (accessed 9 August 2019).

6. National Academy of Sciences USA (2007). Green Schools: Attributes for Health and Learning. Washington: National Academy of Sciences USA.

7. ZVEI (2018). Guide to Human Centric Lighting (HCL). Licht.wissen 21. Frankfurt am Main: ZVEI.

8. ZVEI (2014). Impact of Light on Human Beings. Licht.wissen 19. Frankfurt am Main: ZVEI. 